\title{
Creativity in Design Engineers: Attitudes, Opinions and Potentially Influential Factors
}

\author{
Paul Neufeld ${ }^{I}$, Omid Mirzaei ${ }^{I}$, Mark Runco ${ }^{2}$, and Sean Maw ${ }^{I}$ \\ ${ }^{1}$ University of Saskatchewan and ${ }^{2}$ University of Georgia \\ sean.maw@usask.ca
}

\begin{abstract}
Is creativity important in engineering design? If it is, then why do most undergraduate engineering programs spend so little time teaching creativity? And therefore, as a result of our programs, do our students emerge more creative, less creative or no different compared to when they arrived? If creativity is worth developing, can we accurately measure it in our students, and can we enhance it systematically?

These were some of the questions that motivated the initiation of a creativity research program in the College of Engineering at the University of Saskatchewan. The assumption was that creativity is important in engineering, especially in design. The intent was to understand how we could assess creativity in our students and then enhance it.

The focus of this initial study is a precursor to many of these more applied questions. We had students and faculty from a variety of Colleges, including Engineering, answer an online survey that probed attitudes towards creativity, respondent personality characteristics, opinions regarding conditional influences on creativity, and potential demographic factors influencing the creativity of individuals. As well, we employed a validated creativity attitudes and beliefs measurement tool (rCAB) as an accepted benchmark for assessment.

The survey included both closed- and open-ended questions. The results from some of the open-ended questions have been analyzed to determine emerging groups of similar types of answers, and then efforts have been made to relate the groups in a meaningful framework.

The results for the Engineering students are emphasized, but they are also compared with students and faculty from other Colleges. Closed questions were analyzed using inferential statistical tests (distributions, means, standard deviations, t-tests, ANOVA, Cronbach's alpha), while the open-ended responses are compared more qualitatively when they cannot be quantified easily.

The survey went through ethics approval and was distributed in the latter half of the Fall 2015 term.
\end{abstract}

Keywords: creativity, design, attitudes, opinions, influential factors, survey

\section{INTRODUCTION}

Innovative developments in technology often depend on engineers for success, and innovation depends on creativity $[3,10]$. However, not all types of engineering activities depend on creativity. Indeed, many engineering activities are quite conservative and depend on proven best practices for both efficiency and safety. This begs the question, to what extent are engineers creative? Moreover, to what extent do engineers value creativity?

Recently, a research program in creativity and innovation was initiated in the College of Engineering at the University of Saskatchewan. It was founded on the premise that creativity is fundamental to good design in all innovative contexts. It was driven by the question of whether we are enhancing creativity in our students, inhibiting it, or not affecting it at all. And it was motivated by the belief that creativity can be taught and therefore enhanced.

The contemplation and study of creativity has a long history, going back to the time of Aristotle [22]. In modern times, it has become a subject of much study and speculation, given its importance in technological change and innovation, which is vital to economic growth [23].

Creativity generally has the connotation of novel and/or original ideation $[17,14,12,19,5]$. Often it is also associated with the realization of such ideas, although we will leave that definition to the domain of innovation [2]. The literature falls largely into two camps regarding the origins of creativity. One camp sees it as a personality trait [16] i.e. you are creative or you are not. The other sees creativity as malleable, either through changes in the person and/or in the environment [8], such as in the case of the creative state of a person e.g. I'm feeling creative right now. A third and less popular perspective is that creativity is a skill [12]. A new perspective that we are introducing to the literature is that creativity is a combination of state, trait and skill, much like athletic or artistic abilities. In sports and the performing arts, it is commonplace to regard such abilities as a function of natural ability (trait), practice (skill), and situation (state).

Factors thought to influence creativity are many but they can be broadly categorized as either individualistic, 
group/team, or organizational. Variables such as team diversity [7], personality characteristics [4, 9, 13, 16, 20], and culture $[15,18]$ can have a bearing on creative expression. The thought processes associated with "being creative" are often characterized as divergent (coming up with many ideas), although convergent (seeing the forest for the trees) may be just as important [6], especially for engineers. Researchers have also looked at how creative thinkers organize their thoughts and ideas, and the steps they go through to "be creative". There has been much study on thinking processes such as incubation [11] and fixation $[25,21]$, which are both highly relevant to design.

Ultimately, if we believe that we can enhance, diminish, gain and/or lose creativity, we will need a way to measure it. Such psychometric tools exist. They are based on presumptions about what constitutes creativity, and about how creativity works. Thus, the field is fraught with controversy and debate. Our research group is invested in the development of new tools to assess creativity, based on our state/trait/skill model. However, this paper focuses on the step before assessment.

This paper documents the first steps in our exploration of creativity. We begin with the most fundamental of questions. What is creativity? Who values it, why do they value it, and how much do they value it? What factors influence and correlate with affinity for creativity? We asked most community members of the University of Saskatchewan these types of questions in the Fall of 2015, and after receiving over 2000 responses, we are just beginning to unravel the answers to these questions. We will highlight the answers from the more than 200 engineers that responded, in comparison to the broader campus population, in hopes of starting to characterize our College's characteristics as they pertain to attitudes and values regarding creativity.

\section{METHODS}

An online pilot survey was distributed to students and faculty from a variety of Colleges, including Engineering, at the University of Saskatchewan during the Fall 2015 term. Ethics clearance was secured from the University of Saskatchewan Behavioural Research Ethics Board. Both open and closed questions were used in this survey with no limits for answers to open-ended questions in terms of response length. Survey questions examined respondents' affinity for creativity, their personality characteristics, their opinions on state, trait and skill-based viewpoints on creativity, and demographic details.

The survey started with a validated Creative Attitudes and Values measurement tool (part of the Runco Creativity Assessment Battery $(\mathrm{rCAB})^{\odot}$ 2012). The Creative Attitudes and Values tool consists of 25, 5-point Likert scale items. Out of these 25 items, 15 and 10 were indicative and contraindicative items, respectively. Contraindicative items were reverse coded and were used along with the indicative ones. Both past research and our current results show good inter-item reliability scores for this measurement tool (see Results).

After this measure, we asked a general question about personal definitions of creativity. This was followed by a question about the nature of creativity. Based on our hypotheses, we gave respondents three choices that corresponded to the three distinctly different viewpoints on creativity i.e. state, trait, and skill. For state, we had "People have creative moments i.e. sometimes they are in a creative mood, and sometimes they are not". For trait, we had "Some people are naturally creative and some people are not". And lastly, for skill, we had "People are more creative when they work at it and practice it, and less so when they don't'. Respondents were able to select as many of the choices as they agreed with i.e. they could indicate that they thought creativity was one element or any combination of these three elements. Later on, we asked the same question again to test whether the survey itself affected their original viewpoint on creativity.

After these initial "nature of creativity" questions, nine word pairs were presented (e.g. optimistic/pessimistic), and respondents rated where their personality fell on that spectrum using a 7-point Likert scale. We then asked further personality-based questions using 5-point Likert scales.

In the next block of questions, we probed opinions on creative behavior (e.g. Is being creative generally a positive behavior?) and solicited open responses on why they felt that way. We also asked whether their opinion had changed since starting their post-secondary education, again letting them explain how (if it had changed). Through this self-assessment, we hope we can see trends in changing attitudes toward creativity on our campus.

Then a number of open-ended questions were asked such as: "If you feel that there are specific situations where it is difficult to be / it is easy to be / one should be / one should not be creative, please list them". Up to two answers could be recorded for each case. These questions were asked to see whether respondents believed in a statebased conceptualization, by decomposing the concept of "state". Likewise, two other questions were asked to assess creativity as a skill i.e. "In your opinion, can you enhance/grow your levels of creativity?" and "can you lose/diminish your ability to be creative?" For these yes/no questions, they could also list two examples supportive of their position. All in all, the answers to these six questions ensured that their definitions were captured, even if the respondents' definitions of a behavioral state and a skill were not consistent with conventional definitions.

The last block of questions asked for demographic information such as academic status, program, year of study (only if the respondent was a student), age, gender, language literacy, favourite childhood games/toys, main pastimes/hobbies, children (whether they had any or not), religious affiliation, and high school origin (urban, rural, or 
international). The literature points out family background as an influential factor on creativity [24]. Therefore, family background questions were asked such as: what is your birth order, how many siblings do you have, what was your family's economic status when you were growing up, and what is the highest level of education that either of your parents has completed. These questions help us to examine effects that originate from family backgrounds and personality shaping factors, and to identify other variables explaining attitudes and values about creativity over and above these other factors.

The results presented in this paper cover the first and most basic level i.e. question-by-question results, as necessitated by time constraints and space limitations for this publication. Excel ${ }^{\mathrm{TM}}$ and SPSS $^{\mathrm{TM}}$ were used to conduct the analyses for this study. All closed questions were analyzed for this paper. Two straight-forward open questions were also analyzed using rigorous grounded theory and organic coding of responses. A preliminary review of this qualitative data has shown some interesting findings and these will be reported in the Results section. The main focus at present is to test whether there is any significant differences in $\mathrm{rCAB}$ scores between different groups of respondents specified by various factors.

\section{RESULTS}

More than 2000 surveys were collected online, as a result of soliciting responses from several of the major Colleges at the University of Saskatchewan. This represents approximately $9 \%$ of the total students, faculty, and academic staff at the University (pop. > 22,000). Figure 1 shows the relative distribution of total responses received from each of the Colleges that participated.

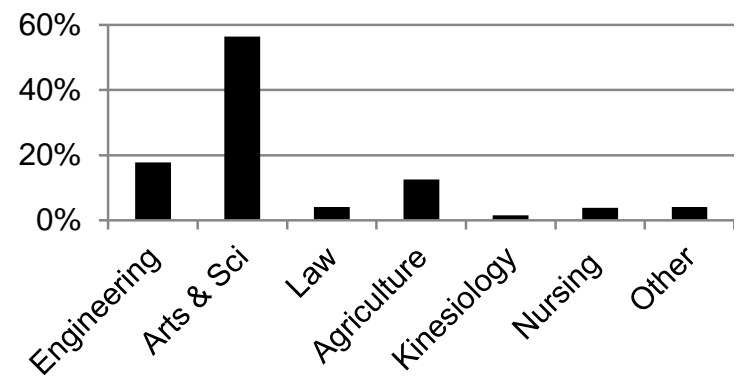

Fig. 1 Percentage of survey respondents by College

Our sample was quite representative of the University population. Respondents from the College of Arts and Science made up the largest cohort for our survey (56.5\%). Arts and Science is also the largest College on campus, constituting approximately $43 \%$ of the total student enrollment population. College of Engineering (CoE) respondents made up $17.8 \%$ of our survey responses, and the $\mathrm{CoE}$ accounts for approximately $10 \%$ of the University's student headcount. From all of the respondents, 1234 of the survey responses were complete, while 781 were incomplete and were used whenever it was possible to do so for data analysis. Only 2 of the survey responses were completely excluded from any of the analyses.

From the perspective of the affiliation with the university, $72 \%$ of respondents were undergraduates, $12 \%$ were in graduate studies, $8 \%$ were faculty/instructors, and another $8 \%$ were staff. Specific to the CoE, the distribution was $73 \%, 16 \%, 6 \%$, and $5 \%$, respectively.

In terms of gender, $61 \%$ of respondents were female and $38 \%$ were male. These numbers are close to the demographics of the student population at the University, which is $57 \%$ and $43 \%$, respectively. Approximately $1 \%$ of the respondents identified their gender as "other", which is a higher representation in our survey responses as compared to the $0.1 \%$ of total students who do not identify as male or female. When it comes to the gender of the completed engineering responses, the breakdown is $30 \%$ female, $69 \%$ male, and $1 \%$ other. The current percentages in the $\mathrm{CoE}$ are $20.7 \%, 79.2 \%$, and $0.1 \%$, respectively. It should be noted that only $61 \%$ of all the respondents actually identified their gender in the survey.

The mean age of all survey respondents was 26.4. The minimum age of all respondents was 17, while the maximum was 76 . Fifty percent $(50 \%)$ of the respondents were 21 or younger, and $80 \%$ of the respondents were 30 or younger. For the CoE, the mean age was 24.5. The $\mathrm{min} / \mathrm{max}$ ages were 17 and 59 . Over $50 \%$ of the CoE respondents were 21 or younger, and $86 \%$ were 30 or younger.

In terms of academic programs within the CoE, Figure 2 shows the response distribution based on each of the programs identified. Note that students not yet registered in a specific department (i.e. first year students) are accounted for in "General" while non-program members (i.e. faculty and staff) and blank program responses, were put in the "Other" grouping.

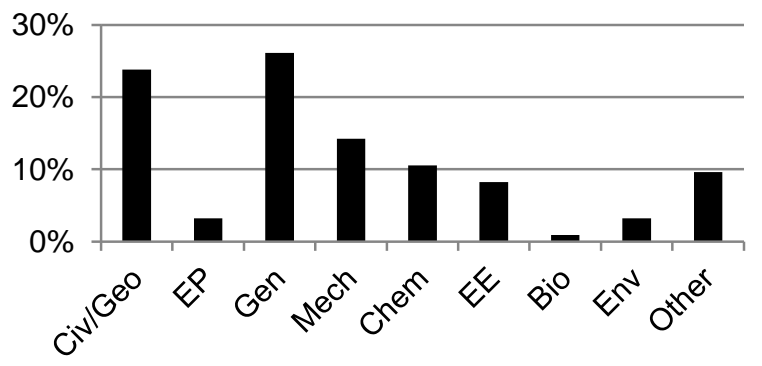

Fig. 2 Percentage of CoE respondents by Program

One of the key parts of the survey was the validated assessment questions from the Runco Creativity Assessment Battery (rCAB) ${ }^{\odot} 2012$ having to do with attitudes and values towards creativity. We will refer to the responses to these questions as the $\mathrm{A} \& \mathrm{~V}$ scores. The 
purpose of the $\mathrm{A} \& \mathrm{~V}$ questions was to try and assess a person's attitudes and values about creativity, their tendencies to act creatively, and their affinity for creativity. Attitudes can greatly influence the probability of thinking and feeling in a creative fashion. Values concerning creativity are incorporated in the score as well, to indicate how much a person appreciates divergence, originality, autonomy, etc. Attitudes and values are both predictive of actual behavior [1]. By completing these questions as part of the survey, we effectively captured an A\&V score (X) for each respondent, based on the entire set of questions. We also calculated an $A \& V$ score using only the positive indicator questions $\left(\mathrm{X}_{\mathrm{pos}}\right)$, omitting responses to the contraindicative questions. To confirm the reliability of using the $A \& V$ questions as part of our survey and subsequent analysis of results, we calculated Cronbach's alpha $(\alpha)$ scores, which were 0.76 and 0.77 , respectively. Therefore, we consider the test reliable for use i.e. $\alpha>0.7$. When looking at the statistics for the $\mathrm{A} \& \mathrm{~V} \mathrm{X}_{\text {pos }}$ scores, the following table shows the statistics and differences between the scores for all non-engineering respondents, and those for just the CoE respondents.

Table 1 Statistics and Comparison of A\&V $X_{\text {pos }}$ scores (Non-Engineering vs. CoE)

\begin{tabular}{|c|c|c|}
\cline { 2 - 3 } \multicolumn{1}{c|}{} & \multicolumn{2}{c|}{ A\&V $\left(X_{\text {pos }}\right)$} \\
\cline { 2 - 3 } & $\begin{array}{c}\text { Non- } \\
\text { Engineering }\end{array}$ & CoE \\
\hline Mean & 45.0 & 45.3 \\
\hline Max. & 60 & 57 \\
\hline Min. & 10 & 27 \\
\hline Std. Dev. & 6.1 & 5.5 \\
\hline
\end{tabular}

From this table, it can be seen that there are marginal differences in the statistics of $\mathrm{A} \& \mathrm{~V} \mathrm{X}_{\text {pos }}$ scores between non-engineers and engineering respondents. While there is a large disparity in the minimum score, the remaining statistics are fairly close to each other in value.

For the remainder of the results, we will focus on the analysis of the data collected from the CoE respondents.

Questions 2 and 14 in the survey were the repeated question about the nature of creativity i.e. is it a trait, state, skill, or some combination? The baseline response was Question 2. Figure 3 shows the initial responses collected from the engineering respondents, and how their opinions changed (if at all) during the survey, as reflected in their response to Question 14.

Over $90 \%$ of the CoE respondents initially felt that creativity was some combination of a trait, state, or skill; $43 \%$ felt that it was a combination of all three. While many respondents did not change their opinions between Question 2 and Question 14, what is interesting to note is that of the $8.6 \%$ of respondents who did change their opinion, almost all changed it to identify that they believed creativity was indeed a combination of trait, state, and skill. The data for Question 14 showed that in the end, 50\% of the engineering respondents felt that creativity was a combination of a trait, state, and skill.

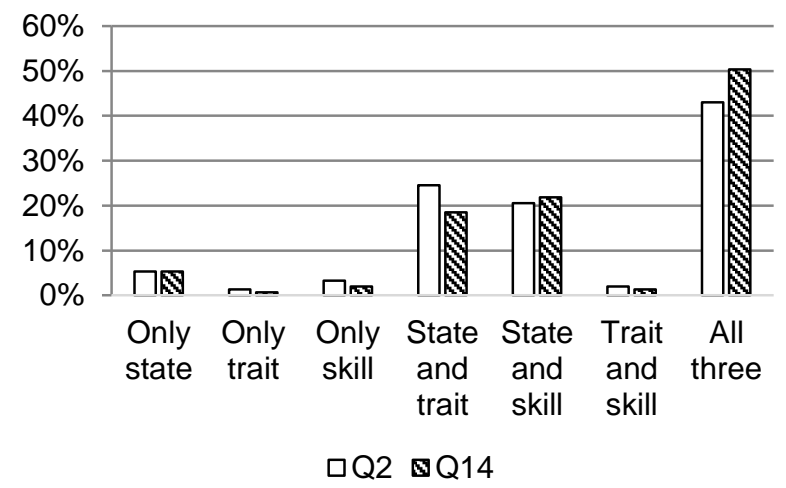

Fig. 3 Initial Opinions of CoE on Creativity and Changing Opinions through Completion of the Survey

Another pair of questions that attempted to identify how respondents felt about creativity, and if their opinions had changed while at university, included asking yes/no questions on "Is being creative generally a positive behavior?" (Q6) and "Has your opinion about Question 6 changed since starting your post-secondary education?" (Q7). Figure 4 depicts how the CoE responded to this pair of questions.

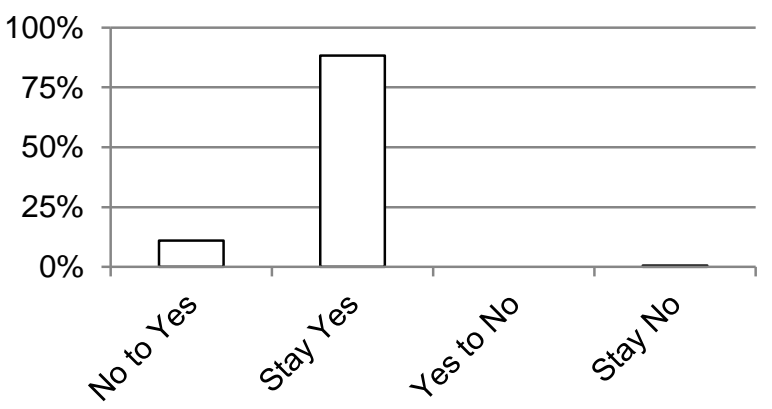

Fig. 4 Differences and Changes in CoE Attitudes towards Creativity for Q6 and Q7

It is apparent that the vast majority (99\%) of engineering respondents now feel that being creative is generally a positive attribute. A full $88 \%$ of those respondents felt that way prior to their post-secondary education and still feel that way, while $11 \%$ have actually started to feel this way only since beginning their postsecondary education.

As part of our analyses, we compared responses to individual questions with the $\mathrm{A} \& \mathrm{~V} \mathrm{X}_{\mathrm{pos}}$ score for each respondent. For this paper, we focus on results which show statistical significance. Note that there was no significance found between the $\mathrm{A} \& \mathrm{~V} \mathrm{X}_{\text {pos }}$ scores and the demographic 
information previously noted, such as gender or age. However, other questions did show significant differences.

Question 3 of our survey identified several word pairs, and asked respondents to make a choice along a 7-point Likert scale that best represented their personality's balance between those two words/phrases. Figures 5 and 6 identify two particular word pairs of interest. Each of the figures shows the average $\mathrm{A} \& \mathrm{~V} \mathrm{X}_{\mathrm{pos}}$ scores for groups with similar responses, along with the standard deviation of the group. The percentage of total respondents in that group is also depicted, in grey.

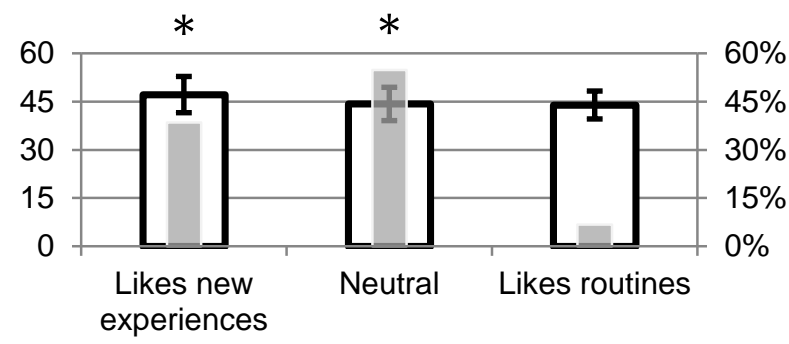

口A\&V Xpos Avg. $\quad \%$ of Respondents

Fig. 5 A\&V Xpos vs. Word Pair Responses (likes new experiences vs. likes routine). * significant difference at $90 \%$ confidence level i.e. $\alpha=.1$

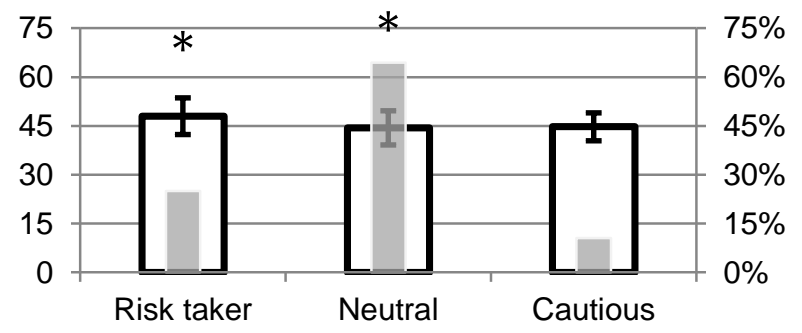

DA\&V Xpos Avg. $\quad \%$ of Respondents

Fig. 6 A\&V $X_{\text {pos }}$ vs. Word Pair Responses (risk taker vs. cautious). *significant difference at $90 \%$ confidence level

In these figures, "neutral" represents the middle three responses on the 7-point Likert scale, while the pair of responses at the left end of the scale represents the left word/phrase and the pair of responses at the right end of the scale represents the right word/phrase. For both word pairs shown, the majority of the respondents were neutral. However, for the respondents that identified as "liking new experiences" and/or being a "risk taker", they exhibited the highest average $\mathrm{A} \& \mathrm{~V} \quad \mathrm{X}_{\mathrm{pos}}$ scores (47.2 and 48.0, respectively) of all the groups responding.

Given our background and context, it may not be surprising that the main focus of our research program on creativity is to assess the levels of these qualities among engineering students, and to then enhance them. In our survey, Question 4 was another Likert scale question (5 point), asking respondents to identify how well various statements described them. The statements of "I am a creative person" and "I am a curious person" yielded interesting results as part of our analyses for $\mathrm{CoE}$ respondents. The results are shown in Figures 7 and 8, respectively.

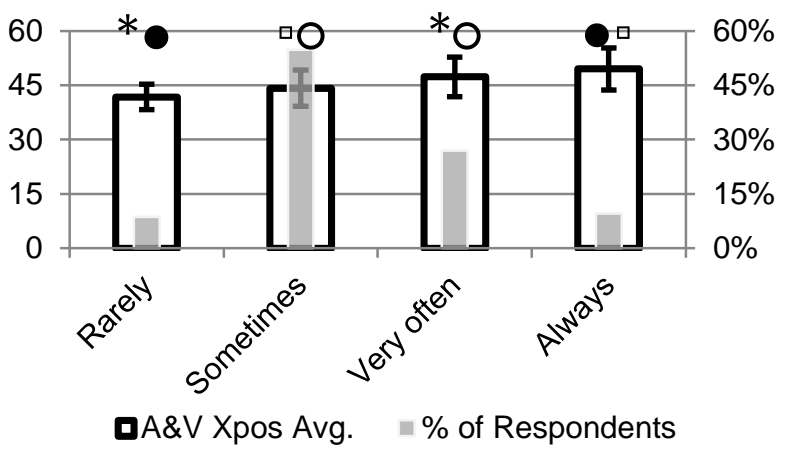

Fig. 7 A\&V Xpos vs. Response to "I am a creative person". ${ }^{*}, \bullet$, , , and $\circ$ indicate significant differences at $90 \%$ confidence level

When responding to the statement "I am a creative person", none of the CoE respondents felt that they were "never" creative, while only $8 \%$ felt that they were "rarely" creative. The majority $(55 \%)$ felt that they were "sometimes" creative, although a significant minority $(37 \%)$ felt they were either "very often" or "always" creative.

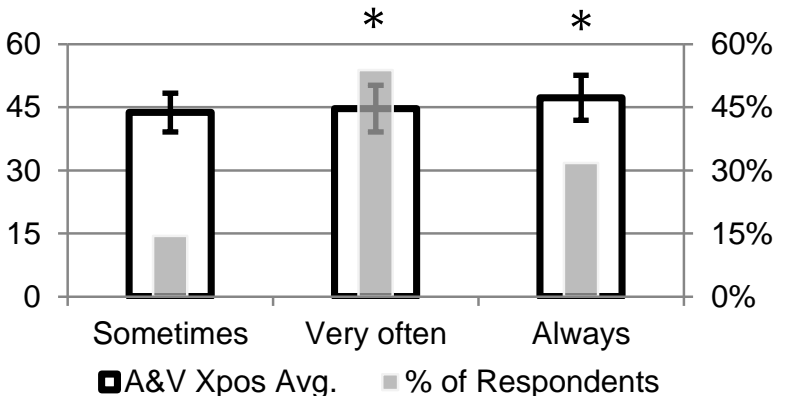

Fig. $8 \mathrm{~A} \& \mathrm{~V} \mathrm{X}_{\text {pos }}$ vs. Response to the statement "I am a curious person". "significant difference at $90 \%$ confidence level

For the statement "I am a curious person" the smallest percentage $(14 \%)$ of respondents chose "sometimes" as their response, while no respondents chose "never" or "rarely". For this question, the majority (54\%) indicated that they were curious "very often". What is interesting to note in both figures is that there was a monotonic increase in $\mathrm{A} \& \mathrm{~V} \mathrm{X}_{\text {pos }}$ scores (attitudes about creativity) with increasing self-assessments of being creative and curious.

As part of our research on curiosity and creativity, we also want to investigate if and how both may be sensitive to environmental influences. As such, we examined which toys, games, hobbies and pastimes were dominant in respondents' lives. In our survey, we asked all respondents to identify their top two pastimes/hobbies as well as their top two favourite toys/games from their childhood. We 
categorized the qualitative responses into several major groupings, codified the data set, and removed any redundancies where a respondent identified more than one example in the same grouping or sub-grouping. By organizing the data in this way, we were able to compare the responses to these questions against the $\mathrm{A} \& \mathrm{~V} \mathrm{X}_{\text {pos }}$ scores of each individual, thus arriving at statistics for the $\mathrm{A} \& \mathrm{~V} \mathrm{X}_{\text {pos }}$ scores of each respondent grouping. Table 2 shows the percentage of $\mathrm{CoE}$ respondents, and the average and standard deviation of the $\mathrm{A} \& \mathrm{~V} \mathrm{X}_{\text {pos }}$ score, for pastime/hobby groups (Q30) that at least $2.5 \%$ of respondents identified with (note: only 4 of 13 groupings did not have more than $2.5 \%$ of total respondents identifying a pastime or hobby as part of the group).

Table 2 A\&V X $X_{\text {pos }}$ vs. Favourite Pastimes/Hobbies

\begin{tabular}{|c|c|c|c|}
\hline \multirow[b]{2}{*}{ Favourite Pastimes/Hobbies } & \multirow[b]{2}{*}{$\begin{array}{c}\% \text { of } \\
\text { Respondents }\end{array}$} & \multicolumn{2}{|c|}{$A \& V\left(X_{\text {pos }}\right)$} \\
\hline & & Avg. & Std. Dev. \\
\hline 1.Playing Sports/Outdoor Rec & $35 \%$ & 45.9 & 4.9 \\
\hline 2. Making/Writing Music & $4 \%$ & 46.1 & 5.2 \\
\hline 3. Doing Other Arts & $17 \%$ & 46.1 & 3.9 \\
\hline 4. Collecting/Hobbies/Interests & $7 \%$ & 46.7 & 5.1 \\
\hline 5. Reading & $10 \%$ & 45.9 & 7.3 \\
\hline 6. Socializing & $3 \%$ & 45.0 & 5.4 \\
\hline 7. Farming/Pets/Animals & $4 \%$ & 44.9 & 5.0 \\
\hline 9. Watching/Appreciating & $8 \%$ & 46.8 & 4.4 \\
\hline 13. Games & $9 \%$ & 44.8 & 7.0 \\
\hline
\end{tabular}

Table 2 shows that the most popular pastime/hobby of the CoE respondents was "Playing Sports/Outdoor Rec" (35\%). The grouping that had the largest average A\&V $\mathrm{X}_{\text {pos }}$ score was that of "Collecting/Hobbies/Interests". This grouping contained respondents who enjoyed everything from cars and home renovations, to collecting stamps. Of the various pastime/hobby groups, four accounted for over $70 \%$ of the total responses from the CoE.

As for the top two favourite toys/games (Q23) from one's childhood, initial results confirm what many engineers have long suspected. Engineers (at U of S, at least) are much more likely to have enjoyed playing with "construction" type toys (e.g. Lego ${ }^{\mathrm{TM}}$ ) in their childhood than non-engineers, as well as video games, puzzles and sports activities. Figure 9 shows the distribution of most favoured toys/games between engineering respondents and non-engineering respondents.

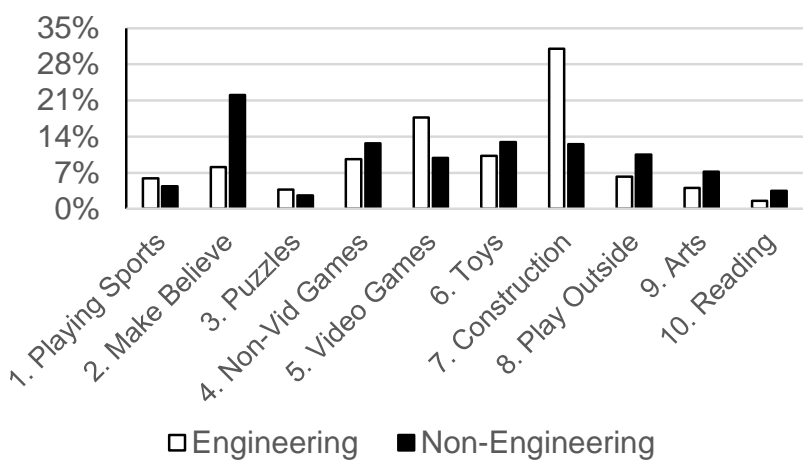

Fig. 9 Percentage of respondents by type of favourite childhood toy/game.

The final question in our survey (Q31) was another demographic question, which asked respondents to identify if they had children or not. What is interesting is that while the vast majority $(91 \%)$ of $\mathrm{CoE}$ respondents indicated that they did not have children, for the $9 \%$ that did identify as having children, the average $\mathrm{A} \& \mathrm{~V} \mathrm{X}_{\text {pos }}$ score for this group was significantly larger than the other group (see Fig. 10).

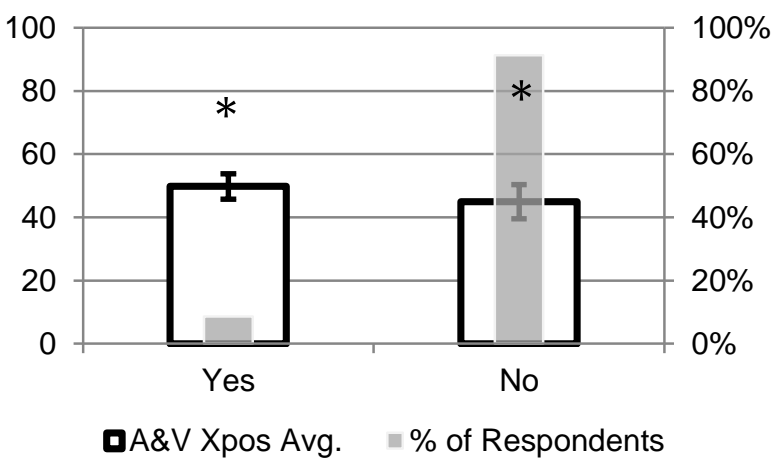

Fig. $10 \mathrm{~A} \& \mathrm{~V} \mathrm{X}_{\text {pos }}$ vs. Do you have any children (Q31)? * indicates significant differences at $90 \%$ confidence level

\section{DISCUSSION}

Currently, most of the lessons learned in this study have been methodological as opposed to results-driven. We have only just scratched the surface of the data analyses.

While not an easy process, coding of text responses has proven to be a very efficient way of analyzing large text data sets. When faced with uncertainty as to what factors are important in an investigation, open-ended questions are effective at casting a wide net that can help inform and enhance the validity of future work. For example, in the case of pastimes and hobbies, it will be possible to gather more refined data in future surveys. Interpretation of current responses were made that may not always be correct. When a respondent said that "art" was a favourite pastime or hobby, did they mean watching/appreciating art or creating art? We cannot know for certain. However, future versions of this survey can offer a finite number of choices to respondents (along with an "Other" category to 
catch unanticipated responses). These can help achieve a precision and validity in the responses that open-ended questions cannot, but these more precise lines of inquiry are often only possible after judicious open-ended questioning, such as what we have engaged in.

It is also vital to get enough data from the demographics that are of concern, in order to drill down and still have statistically valid results. We do have a large amount of data, but perhaps not enough from engineering to provide as much insight, ultimately, as we would have hoped for this group. In other words, the distribution of data is as important as the volume of it.

In the use of Likert scales, it has proven valuable to aggregate leftmost, rightmost, and central responses on 5point or 7-point scales. This tactic addresses the reluctance of some people to make extreme choices, although they hold strong views. It also helps capture those with views so close to neutral that they are effectively neutral. It is, in a sense, the application of a low-pass filter on Likert scales.

\section{CONCLUSIONS AND FUTURE WORK}

If the University of Saskatchewan community is any measure, then many people regard creativity as a personal quality that is some combination of trait, state and skill, much like athletic and artistic abilities. This is even more so the case when people are introduced to the prospect of such a possible definition.

The initial analyses of our data suggest that university does not diminish the value that we attach to creativity. And it would appear that certain personality characteristics correlate with positive attitudes towards creativity. These include a preference for new experiences, and risk taking. Those who believe that they are creative and curious do seem to have more positive attitudes towards these qualities. No clear pattern has emerged yet, regarding the correlation of pastimes and hobbies with valuing creativity. However, having children seems to correlate with people regarding creativity more favourably. And engineers do seem to have enjoyed playing with construction-type toys in their childhood, more than most other disciplines.

Our data set is quite large, and the analyses of the data are only in the preliminary stages. Coding of the text-based answers is the top priority at this point, so that more rapid quantitative analyses can follow. These should and will include all cross-tabs of interest, to see how responses interact. Of particular interest to us are the engineering responses, of course. However, they are too few in number to allow too deep an examination with any statistical significance. As such, a high priority will be to secure additional data from other engineering schools, if possible. This will take place after the coding of the text responses from the current survey, so that more accurate and valid measures can be garnered from future data collections.
Attention will also be devoted to finding and/or developing effective psychometrics that can assess creative abilities, given our tripartite perspective on them.

\section{Acknowledgements}

The authors would like to thank Don Listwin, funder of the Huff Chair at the University of Saskatchewan, for his vital support. Huff Chair research funding made this work possible. As well, we deeply appreciate the support of the Deans of Engineering, Kinesiology, Arts \& Science, Medicine, Nursing, Agriculture, and Law, at the University of Saskatchewan, for letting us distribute the survey in their Colleges. Finally, we want to thank Jade Knoblauch and the members of InTREEg (Innovative Teaching and Research in Engineering Education Group) this past year for their help and input during the development of this study.

\section{References}

[1] S Acar and M. A. Runco, "Assessing associative distance among ideas elicited by tests of divergent thinking," Creativity Research Journal, pp. 229-238, 2014.

[2] T M Amabile, "A model of creativity and innovation in organizations," Research in Organizational Behaviour, pp. 123-167, 1988.

[3] Jamie D Barrett et al., "Thinking About Applications: Effects on Mental Models and Creative Problem-Solving," Creativity Research Journal, pp. 199-212, 2013.

[4] Sarah White Bender, BradyLeigh Nibbelink, Elizabeth Towner-Thyrum, and Debra Vredenburg, "Defining Characteristics of Creative Women," Creativity Research Journal, pp. 38-47, 2013.

[5] Fa-Chung Chiu, "Fit between future thinking and future orientation on creative imagination," Thinking Skills and Creativity, pp. 234-244, 2012.

[6] Arthur Cropley, "In Praise of Convergent Thinking," Creativity Research Journal, pp. 391-404, 2006.

[7] Petru Lucian Curşeu, "Team Creativity in Web Site Design: An Empirical Test of a Systemic Model," Creativity Research Journal, pp. 98-107, 2010.

[8] Dan Davies et al., "Creative learning environments in education-A systematic literature review," Thinking Skills and Creativity, pp. 80-91, 2013.

[9] Candice D Davis, James C Kaufman, and Faith H McClure, "Non-Cognitive Constructs and Self-Reported Creativity by Domain," The Journal of Creative Behavior, pp. 188-202, 2011. 
[10] Mónica Edwards-Schachter, Ana García-Granero, Mabel Sánchez-Barrioluengo, Henry Quesada-Pineda, and Nabil Amara, "Disentangling competences: Interrelationships on creativity, innovation and entrepreneurship," Thinking skills and creativity, pp. 27-39, 2015.

[11] Sophie Ellwood, Gerry Pallier, Allan Snyder, and Jason Gallate, "The Incubation Effect: Hatching a Solution?," Creativity Research Journal, pp. 6-14, 2009.

[12] Anusca Ferrari, Romina Cachia, and Yves Punie, Innovation and Creativity in Education and Trainingin the EU Member States: Fostering Creative Learning and Supporting Innovative Teaching - Literature review on Innovation and Creativity in E\&T in the EU Member States (ICEAC). Luxembourg: Office for Official Publications of the European Communities, 2009.

[13] Adrian Furnham, David J Hughes, and Emma Marshall, "Creativity, OCD, Narcissism and the Big Five," Thinking Skills and Creativity, pp. 91-98, 2013.

[14] Panagiotis G Kampylis and Juri Valtanen, "Redefining Creativity - Analyzing Definitions, Collocations, and Consequences," The Journal of Creative Behavior, pp. 191214, 2010.

[15] Lan Lan and James C Kaufman, "American and Chinese Similarities and Differences in Defining and Valuing Creative Products," The Journal of Creative Behavior, pp. 285-306, 2012.

[16] Diane Montgomery, Kay S Bull, and Lynda Baloche, "Characteristics of the Creative Person Perceptions of University Teachers in Relation to the Professional Literature," American Behavioral Scientist, pp. 68-78, 1993.
[17] Michael D Mumford, "Where have we been, where are we going? Taking stock in creativity research," Creativity Research Journal, pp. 107-120, 2003.

[18] Weihua Niu and James C Kaufman, "Creativity of Chinese and American Cultures: A Synthetic Analysis," The Journal of Creative Behavior, pp. 77-87, 2013.

[19] Davide Piffer, "Can creativity be measured? An attempt to clarify the notion of creativity and general directions for future research," Thinking Skills and Creativity, pp. 258264, 2012.

[20] Meihua Qian, Jonathan A Plucker, and Jiliang Shen, "A Model of Chinese Adolescents' Creative Personality," Creativity Research Journal, pp. 62-67, 2010.

[21] Steven M Smith and Julie Linsey, "A Three-Pronged Approach for Overcoming Design Fixation," The Journal of Creative Behavior, pp. 83-91, 2011.

[22] John Alexander Smith and William David Ross, The works of Aristotle. Oxford: Clarendon press, 1912.

[23] Robert M Solow, "A Contribution to the Theory of Economic Growth," The Quarterly Journal of Economics, pp. 65-94, 1956.

[24] Hung-Yi Wu, Hung-Shu Wu, I-Shuo Chen, and Hui-Chun Chen, "Exploring the critical influential factors of creativity for college students: A multiple criteria decision-making approach," Thinking Skills and Creativity, pp. 1-21, 2014.

[25] Robert J Youmans, "Design Fixation in the Wild: Design Environments and Their Influence on Fixation," The Journal of Creative Behavior, pp. 101-107, 2011. 